\title{
Agents in Home Care: A Case Study
}

\author{
Juan A. Fraile ${ }^{1}$, Dante I. Tapia², Sara Rodríguez ${ }^{2}$, and Juan M. Corchado \\ ${ }^{1}$ Pontifical University of Salamanca, c/ Compañía 5, 37002 Salamanca, Spain \\ jafraileni@upsa.es \\ ${ }^{2}$ Departamento de Informática y Automática, University of Salamanca, \\ Plaza de la Merced s/n, 37008 Salamanca, Spain \\ \{dantetapia, srg, corchado\} @usal.es
}

\begin{abstract}
Home Care is the term used to refer to any kind of care to a person at his own home. This article presents a case study of the HoCa hybrid multiagent architecture aimed at improving of dependent people in their homes. Hoca architecture uses a set of distributed components to provide a solution to the needs of the assisted people and its main components are software agents that interact with the environment through a distributed communications system. This paper describes the hybrid multiagent system in a home care environment and presents the results obtained.
\end{abstract}

Keywords: Dependent environments, Hybrid Multiagent Systems, Home Care.

\section{Introduction}

"Divide and conquer" is a technique that has been widely used to resolve large and complex problems, where each part of the problem is easier to handle and to find its solution separately. To that end, it is necessary to act cooperatively, and follow some order of execution in the way of communication between tasks. In order to resolve a task it will be necessary to know the type of inputs and outputs expected, as well as distributed computing methods. One of the alternatives is the use of hybrid systems. Multiagent hybrid systems [3] try to combine the runtime reactive agents with the rationality of the deliberative agents. These hybrid multi-agent systems are specially used when the requirements of the problem can not be satisfied by neither reactive multiagents systems nor deliberative multiagent systems independently. The integration of reactive and deliberative agents requires three key concepts: communication, cooperation and coordination. The HoCa architecture focuses in these concepts to facilitate the development of home care environments.

Home Care requires the improvement of the services offered to the users as well as the way they can be accessed [3]. Moreover, it is necessary to adopt the trends already tested and proven in technological environments [1]. Intelligent environments are focused on the user, since the user is the centre of the new technological facilities and demands access to unified services [2]. The importance acquired by the dependency people sector has dramatically increased the need for new home care solutions [5]. Besides, the commitments that have been acquired to meet the needs of this sector, suggest that it is necessary to modernize the current systems. Multiagent systems [14], and intelligent devices-based architectures have been recently explored as supervisor 
systems for health care scenarios [1] [7] for elderly people and for Alzheimer patients [5] [13]. These systems allow providing constant care in the daily life of dependent patients [4], predicting potentially dangerous situations and facilitating a cognitive and physical support for the dependent patient [2]. Taken into account these solutions, it is possible to think that multi-agent systems facilitate the design and development of home care environments [6] and improve the services currently available, incorporating new functionalities. Multi-agent systems add a high level of abstraction regarding to the traditional distributed computing solutions.

The aim of this paper is to present a case study where the HoCa hybrid multiagent architecture is used to develop a multiagent system to monitor the routine tasks of daily life of the patients and detect dangerous situations at home. It is necessary to integrate new technologies into the patient's home to achieve this objective, using techniques of artificial intelligence, intelligent agents and wireless technologies. The purpose is to optimize the effectiveness and management of the home care to facilitate the working conditions of the medical staff and improve the patient's quality of life.

The rest of the paper is structured as follows: Section 2 describes the proposed architecture and agents types to resolve the problem. Section 3 describes a case study to test the architecture and finally, Section 4 presents the results and conclusions obtained.

\section{Agent Types in HoCa}

The HoCa multi-agent architecture uses a series of components to offer a solution that includes all levels of service for various systems [9]. It accomplishes this by incorporating intelligent agents, identification and localization technology, wireless networks and mobile devices [10].

The Agents platform is the core of the architecture and integrates two types of agents as show the Figure 1, each of which behaves differently for specific tasks. The first group of agents is made up of deliberative BDI agents [8], who are in charge of the management and coordination of all system applications and services [5]. However, there are pre-defined agents which provide the basic functionalities of the architecture:

- CoAp Agent: This agent is responsible for all communications between applications and the platform. Manages the incoming requests from the applications to be processed by services. It also manages responses from services to applications. CoAp Agent is always on "listening mode". Applications send XML messages to the agent requesting for a service, then the agent creates a new thread to start communication using sockets.

- CoSe Agent: It is responsible for all communications between services and the platform. The functionalities are similar to CoAp Agent but backwards. This agent is always on "listening mode" waiting for responses of services. Manager Agent indicates CoSe Agent the service that must be invoked.

- Directory Agent. Manages the list of services that can be used by the system. For security reasons, the list of services is static and can only be modified manually, however services can be added, erased or modified dynamically. The list contains the information of all trusted available services. 
- Supervisor Agent. This agent supervises the correct functioning of the agents in the system. Supervisor Agent verifies periodically the status of all agents registered in the architecture by means of sending ping messages.

- Security Agent. This agent analyzes the structure and syntax of all incoming and outgoing XML messages. If a message is not correct, the Security Agent informs the corresponding agent (CoAp or CoSe) that the message cannot be delivered.

- Manager Agent. Decides which agent must be called taking into account the users preferences. Users can explicitly invoke a service, or can let the Manager Agent decide which service is better to accomplish the requested task. Manager Agent has a routing list to manage messages from all applications and services.

- Interface Agent. This kind of agent has been designed to be embedded in users' applications. The requests are sent directly to the Security Agent, which analyzes the requests and sends them to the Manager Agent. These agents must be simple enough to allow them execute on mobile devices, such as cell phones or PDA's.

Figure 1 along with a simple example helps to understand the communication between different types of agents in the architecture. A patient is visited by the medical service due to a feverish that suffers by an infection. The medical service went to the house before the patient's explicit request made through the alerts system. The patient through an application has inserted the alert in the system. The CoAp agent is responsible for registering this information into the system and notifies the supervisor agent. The security agent confirmed the credentials of the user who enters the information and validates the information entered. The supervisor agent at the same time performs two tasks, requests the directory agent you select the service to run to launch the alert and through the interface agent informs the manager agent of operations performed. The CoSe agent runs the service that launches the alert and finally the alert is sent through the reactive agents of the architecture. At all times the manager agent is informed of the steps being taken in the system and is responsible for validating the alert sending through the interface agent to the corresponding reactive agent. Once the patient enters the information into the system, this process seems very laborious and slow is running in a few thousandths of a second.

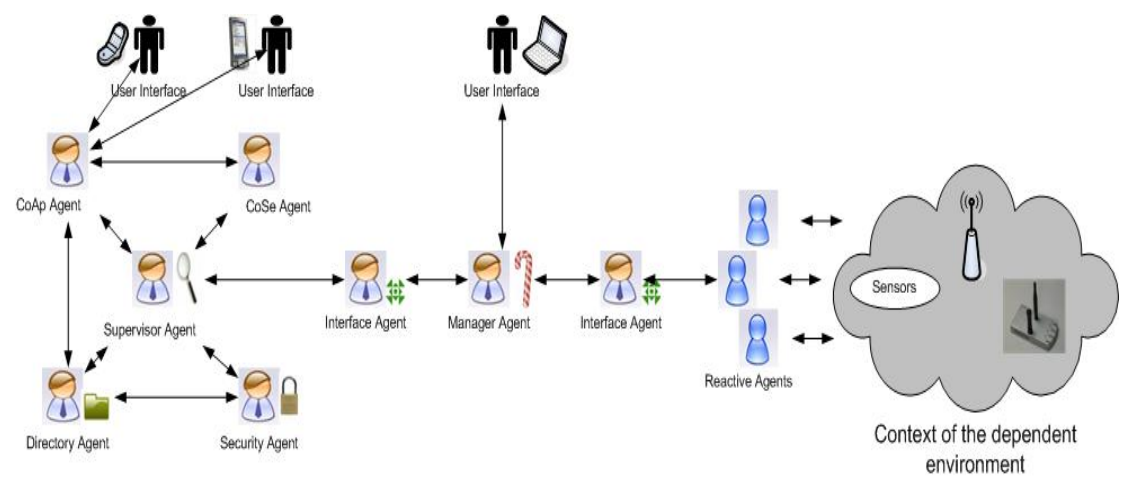

Fig. 1. Agents Workflow in the HoCa architecture 
The second group is made up of reactive agents [4]. Most of the research conducted within the field of multi-agent systems focuses on designing architectures that incorporate complicated negotiation schemes as well as high level task resolution, but don't focus on temporal restrictions. In general, the multi-agent architectures assume a reliable channel of communication and, while some establish deadlines for the interaction processes, they don't provide solutions for limiting the time the system may take to react to events.

\section{Using HoCa to Development a Hybrid Multi-Agent System for Dependent Environment}

HoCa has been employed to develop a hybrid multi-agent system aimed to enhance assistance and care for low dependence patients at their homes. The house has $89 \mathrm{~m}^{2}$ and it live two dependents persons. As shown in Figure 2 are installed 33 passive infrared motion detectors for roof of the SX-360 series and 11 mechanisms for automatic door opening. The detectors movements and mechanisms for opening doors, interact with the microchip Java Card \& RFID [11] users to offer services in run time. Each dependent user is identified by a Sokymat ID bracelet Band Unique Q5 which has an antenna and a chip RFID-Java-Crypto-Card with $32 \mathrm{~K}$ Module and CryptoCoProzessor (1024 bit RSA) compatibel to SUNs JavaCard 2.1.1 [15]. The sensors or actuators are placed in strategic positions from home as shows Figure 2 plane. All these devices are controlled by platform agents. This sensors network through a system of alerts is responsible for generating alarms comparing the user current state with the parameters of the user daily routine who has stored the system. The system can generate alarms if it is determined the parameters for example if the user in a nonworking day stands before a certain hour, or if the user spends more time than specified on the door of your home without entering, or the user is a long time motionless in the hallway, etc.

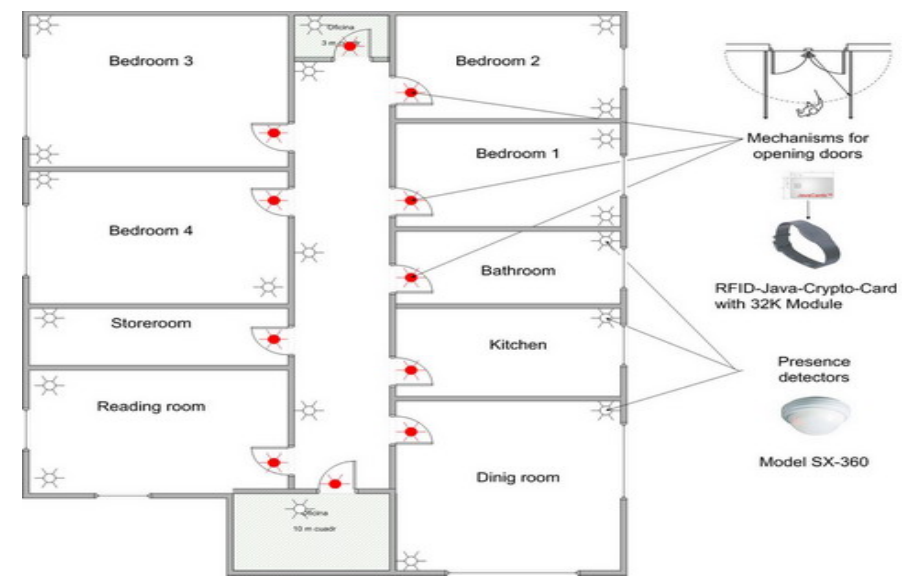

Fig. 2. Home plane 


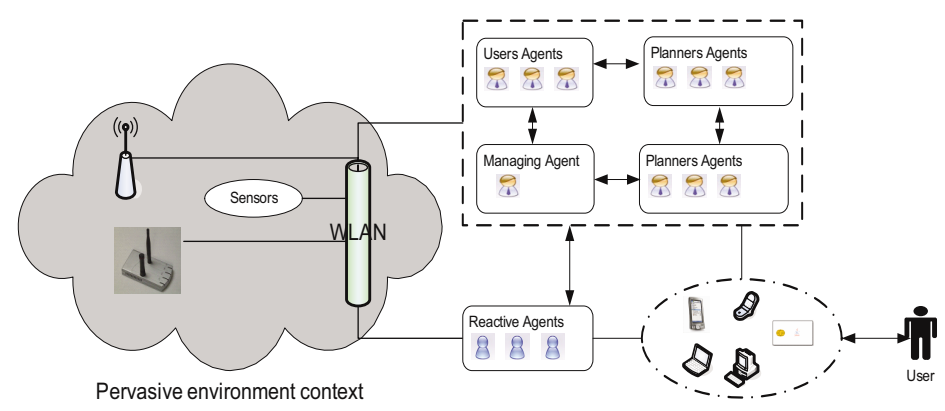

Fig. 3. HoCa structure in a dependent environment

Main functionalities in the system include reasoning, planning mechanisms, management alerts and responses in execution time offered to certain stimuli, as shown in Figure 3. Also in Figure 3 shows as they relate the different types of agents and the connection between the user devices for the execution of services and applications. The pervasive environment there is configured wireless devices and RFID readers also managed by the agents' platform designed. These functionalities allow the system the use of several context-aware technologies to acquire information from users and their environment.

Each agent in the system has its own functionalities. If an agent needs to develop a task in collaboration with other agent a request form is send. There are priority tasks that a set of agents can perform. This ensures that the priority tasks are always available. There are four types of deliberative BDI agents, as shown in Figure 3:

- User Agents. This agent manages the users' personal data and behaviour. The beliefs and goals used for every user depend on the plan or plans defined by the super-users. User Agent maintains continuous communication with the rest of the system agents, especially with the ScheduleUser Agent and with the SuperUser Agent. There is one agent for each patient registered in the system.

- SuperUser Agent. It also runs on mobile devices (PDA) and inserts new tasks into the Manager Agent to be processed by a Case-Based Reasoning mechanism. It also needs to interact with the User Agents to impose new tasks and receive periodic reports, and with the ScheduleUser Agents to ascertain plans' evolution. There is one agent for each supervisor connected to the system.

- SheduleUser Agent. It is a BDI agent with a Case-Based Planning (CBP) mechanism embedded in its structure. It schedules the users' daily activities obtaining dynamic plans depending on the tasks needed for each user. Every agent generates personalized plans depending on the scheduled-user profile. There are as many ScheduleUser Agents as nurses connected to the system.

- Manager Agent. It runs on a Workstation and plays two roles: the security role that monitors the users' location and physical building status trough a continuous communication with the Devices Agent; and the manager role that handle the databases and the tasks assignment. It must provide security for the users and ensure the tasks assignments are efficient. There is just one Manager Agent running in the system. 
On the other hand there are a number of reactive agents that work in collaboration with the deliberative agents. These agents are in change of control devices interacting with sensors (access points, lights, temperature, alarms detection, etc.). They receive information, monitor environment services and also check the devices status connected to the system. All information is treated by the reactive agent and it is sent to the manager agent to be processed.

\section{Results and Conclusions}

The high number of dependent population makes it necessary to develop new approaches to provide specialized care. Technologies such as multi-agent systems, systems for information management, secure communications, multimedia interfaces, domotics or collaborative virtual environments, represent an important factor in the design and development of new home care facilities. In particular, a platform based on agents in combination with mechanisms for information management provides a flexible infrastructure for special care [12]. The collective vision of the HoCa architecture allows the system to create patterns that serve to further responds to the patient's problems.

HoCa architecture has been used in this study to develop a real environment for home care. The multiagent system obtained has been compared to the previous human-based system taking into account the amount of necessary medical staff before and after the implementation of the prototype. Some of the experiments focused on determining the time spent in indirect tasks for patient's care and the number of medical staff working simultaneously monitoring and attending possible emergencies. The results have been satisfactory, showing that the HoCa-based system reduces the response time and optimizes the use of resources. It has been also analyzed the impact of such a system in a real environment, checking the way traditional services can be optimized, enhancing their quality.

Figure 4 shows the comparison of the average medical personnel required in the two studies made with ALZ-MAS and HoCa. The ALZ-MAS architecture [6] allows the monitoring of patients in geriatric residences, but home care is carried out through

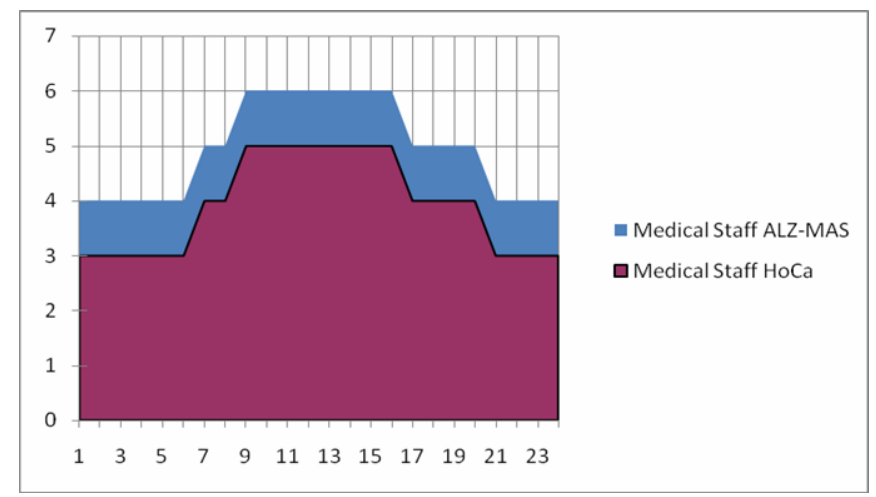

Fig. 4. Comparison of medical staff between the HoCa and the ALZ-MAS architectures 
traditional methods. The case study presented in this work consisted of analysing the functioning of both architectures in a test environment. The HoCa architecture was implemented in the home of 5 patients and was tested for 30 days. Figure 4 represents the number of people who are in need each hour of the day. The graph seen as the medical personnel necessary for both systems is lower during the hours of lesser activity of the patients. On the contrary during the hours of the morning and afternoon will need more health staff. It also looks like the HoCa system does not need as personal as ALZ-MAS.

As the result $\mathrm{HoCa}$ architecture creates an environment that facilitates intelligent and distributed and provides services to dependents at home. Automating tasks and patient monitoring improve the system security and efficiency of care to dependents. The use of RFID technology, JavaCard and mobiles with people provides a high level of interaction between users and patients through the system and is essential in building an intelligent environment. Moreover the good use of mobile devices can facilitate social interactions and knowledge transfer.

\section{References}

1. Angulo, C., Tellez, R.: Distributed Intelligence for smart home appliances. Tendencias de la minería de datos en España. Red Española de Minería de Datos. Barcelona, España (2004)

2. Augusto, J.C., McCullagh, P.: Ambient Intelligence: Concepts and Applications. Invited Paper by the International Journal on Computer Science and Information Systems 4(1), 1-28 (2007)

3. Bajo, J., et al.: Hybrid Architecture for a Reasoning Planner Agent. In: Apolloni, B., Howlett, R.J., Jain, L. (eds.) KES 2007, Part II. LNCS, vol. 4693, pp. 461-468. Springer, Heidelberg (2007)

4. Carrascosa, C., Bajo, J., Julian, V., Corchado, J.M., Botti, V.: Hybrid multi-agent architecture as a real-time problem-solving model. Expert Systems With Applications 34(1), 2-17 (2008)

5. Corchado, J.M., Laza, R.: Constructing Deliberative Agents with Case-based Reasoning Technology. International Journal of Intelligent Systems 18, 1227-1241 (2003)

6. Corchado, J.M., Bajo, J., de Paz, Y., Tapia, D.: Intelligent Environment for Monitoring Alzheimer Patients, Agent Technology for Health Care. Decision Support Systems 34(2), 382-396 (2008)

7. Corchado, J.M., Bajo, J., Abraham, A.: GERAmI: Improving the delivery of health care. IEEE Intelligent Systems. Special Issue on Ambient Intelligence (March/April 2008)

8. Corchado, J.M., Gonzalez-Bedia, M., De Paz, Y., Bajo, J., De Paz, J.F.: Replanning mechanism for deliberative agents in dynamic changing environments. Computational Intelligence 24(2), 77-107 (2008)

9. Fraile, J.A., Tapia, D.I., Sánchez, M.A.: Hybrid Multi-Agent Architecture (HoCa) applied to the control and supervision of patients in their homes. In: Proceedings of HAIS 2008. Hybrid Artificial Intelligence Systems. Third International Workshop, Burgos, Spain, pp. 54-61 (September 2008) ISBN: 978-3-540-87655-7

10. Fraile, J.A., Bajo, J., Pérez, B., Sanz, E.: HoCa Home Care Multi-Agent Architecture. In: International Symposium on Distributed Computing and Artificial Intelligence 2008 (DCAI 2008), pp. 52-61 (October 2008) ISBN: 978-3-540-85862-1 
11. ITAA, Radio Frequency Identification. RFID coming of age. Information Technology Association of America (2004), http: / /www. itaa.org/rfid/docs/rfid.pdf

12. Segarra, M.T., Thepaut, A., Keryell, R., Poichet, J., Plazaola, B., Peccatte, B.: Ametsa: Generic Home Control System Based on UPnP. In: Independent living for persons with disabilities and elderly people, pp. 73-80. IOS Press, Amsterdam (2003)

13. Tapia, D.I., Bajo, J., De Paz, F., Corchado, J.M.: Hybrid Multiagent System for Alzheimer Health Care. In: Proceedings of HAIS 2006. Solange Oliveira Rezende, Antonio Carlos Roque da Silva Filho Eds, Ribeirao Preto, Brasil (2006)

14. Weiser, M.: The Computer for the Twenty-First Century. Scientific American 265, 94-104 (1991)

15. ZhiqunChen (Sun Microsystems). Java Card Technology for Smart Cards. Addison Wesley Longman, ISBN 0201703297 